\title{
The Influence of Complex Distractors in the Remote Distractor Paradigm
}

\author{
Valerie Benson \\ University of Southampton \\ Email: vb1@soton.ac.uk
}

Three experiments examined the influence of complex distractors on the Remote Distractor Effect (RDE), a robust finding of an increase is saccade latencies when two, rather than one possible targets are presented simultaneously (Walker, Deubel, Schneider \& Findlay, 1997). In Experiment 1 saccade onset latencies (SOL's) were greater for central versus peripheral presentation for lexical distractors, but not for non-lexical distractors. Experiment 2 showed that repeated presentation of a distractor results in reduced SOL's at central presentation, regardless of lexical status. In Experiment 3 differences in saccade onsets were obtained between two types of lexical distractor when presented at parafoveal locations. Detailed analysis revealed that although SOL's are modulated by distractor complexity, RDE magnitudes are not.

\section{Keywords: Remote distractor effect (RDE), Complex distractors, RDE magnitudes, Repeated distractor effect}

\section{Introduction}

When two visual stimuli are simultaneously bilaterally presented and a saccadic orienting response is required to one of these stimuli, saccadic latencies are 20$40 \mathrm{~ms}$ slower, compared to when a single target is presented (Lévy-Schoen, 1969). Walker, Kentridge, and Findlay (1995) showed that this increase was not modified by an instruction to attend and saccade to one side of the presentation only. Since it was not affected by a voluntary decision to saccade in a prespecified direction, it was concluded that the bilateral target effect appeared to be an automatic low-level inhibitory effect resulting from competition between two simultaneously presented potential saccade targets.
Further detailed investigation (Walker, Deubel, Schneider \& Findlay 1997) has shown that there is a systematic relationship between the position of the remote distractor and the magnitude of the latency increase, and introduced the term Remote Distractor Effect (RDE) to describe the effect. Centrally presented (foveal) distractors produced the biggest RDE, and the effect decreased systematically as the distractor was positioned more peripherally, out to 10 degrees.

There is still debate over how best to account for the RDE. (e.g. Findlay and Walker, 1999; Godijn and Theeuwes, 2002) but generally the RDE is regarded as a low-level, automated, visual response (although possibly absent if distractors do not activate the magnocellular pathways, Sumner, Adamjee \& Mollon, 2002). What has not yet been examined is whether more complex distractors can produce any systematic effects upon saccade latency in this paradigm. The automaticity of 
lexical processing is well documented (e.g., Stroop, 1935; see also MacLeod, 1991 for a review), therefore one type of complex distractor that could impact upon the RDE could be one that has lexical status. An immediate influence of lexical factors on oculomotor behaviour has been shown during the process of reading and currently, there exists a wealth of data in the reading literature that indicate that lexical variables influence the time it takes to initiate a saccade (Liversedge \& Findlay, 2000: Rayner, 1998).

The types of distractor letter strings to be employed can be categorized within a lexical hierarchy, although it should be noted here that the term lexical in this paper is used only on an operational level, as lexical variables such as word frequency are not manipulated. The simplest lexical string in the set is a uniform repeated letter string (e.g. AAAA). Since such a string is formed from identical constituent letters, it is both visually very simple and minimally lexically complex. On the basis of both visual and lexical characteristics of such strings, categorisation of the string as a nonword should be fast, and consequently, discontinuation of lexical identification procedures should occur quickly and therefore latencies to targets for this type of distractor should be reduced compared to distractors with more complex lexical content. Orthographically illegal nonwords (e.g. LGNT) are comprised of different letters and are therefore more visually and lexically complex than uniform strings. Since such strings violate the rules of orthography that govern words in the English Language, they are categorically dissimilar from words, and again, lexical identification procedures (that may be initiated automatically) should be discontinued after a short time and such letter strings should not engage the language processing system to a great degree. At the next level, orthographically legal nonwords are represented (e.g. LUPT). Such strings are lexically more complex than strings at the preceding level, in that they are orthographically legal and are therefore potentially good lexical candidates. It is merely arbitrary that such strings are not words and therefore do not have a corresponding lexical entry. Finally, the most complex level of lexical string in the hierarchy for these experiments is that of real words. Real words are orthographically legal letter strings that have corresponding lexical entries and therefore semantic meaning (e.g. LOST). Assuming initiation of lexical identification procedures occurs automatically it is anticipated that distractor stimuli from these two levels of the hierarchy should have the greatest magnitude upon the RDE, since they should engage the lexical identification system to the greatest degree (though it is not clear whether lexical processing of a word, or a legal nonword would produce the maximal effect). Using samples of items from each level of this lexical hierarchy provides the opportunity to develop good candidate visual distractor stimuli that permit the systematic investigation of the influence of more complex distractors on low-level oculomotor control.

\section{Experiment 1}

Experiment 1 investigated whether the lexical status of a distractor would systematically affect the magnitude of the RDE, and whether lexical distractors would produce a greater RDE compared to a non-lexical distractor. Distractors were presented at either central (foveal) or peripheral ( 8 degrees eccentric) locations. Participants were required to ignore the distractor and saccade to a target (a cross) presented on half of the trials 8 degrees to the left of the midline of the display, and 8 degrees to the right on the remainder of trials. Since the largest RDEs have been obtained for centrally presented distractors (Walker et al., 1997), and effects of orthographic status have been shown for lexical decision tasks that have presented material at fixation (e.g. Coltheart, Davelaar, Jonasson and Besner, 1977), it was assumed that any effect of lexical status would be most apparent when the strings appeared at central presentation. It has been shown that, at least during the reading of text, only low-level visual information is available to the language processing system outside the parafovea (see Rayner \& Pollatsek, 1989). A reduced effect was, therefore, anticipated for peripherally presented distractors. At the distractor eccentricity used visual acuity is reduced and consequently strings may not be sufficiently visible to permit automatic lexical identification processes (Rayner, Well \& Pollatsek, 1980). 


\section{Method}

\section{Participants}

22 members of the University of Durham acted as volunteers. For all the experiments reported in this paper, participants were native English speakers with normal or corrected to normal vision and were paid to participate. All were naïve to the purpose of the experiments.

\section{Eye movement recording}

Eye movements were monitored using a Fourward Technologies Dual Purkinje Generation 5.5 eye tracker with spatial resolution of $10 \mathrm{~min}$ of arc. Viewing was binocular, but only the movements of the right eye were monitored. Viewing distance was $67 \mathrm{~cm}$. Eye movements were sampled at a rate of $250 \mathrm{~Hz}$.

\section{Materials}

For each of the stimulus files the target was a cross $(+)$, drawn in 4-point plain pen, size $0.8 \mathrm{~cm}$, located at 9.3 $\mathrm{cm}$ ( $8 \mathrm{deg}$ eccentricity) on the left or the right of the midline of the display. On distractor trials, this was accompanied by a distractor string, presented either centrally, or at 8 degrees eccentricity on the opposite side to the target. Both target and distractors were black in colour and the background for each display was white. All characters for the lexical distractor strings were created in upper case text using Helvetica font size 32 point, overall length of string was approximately $3.8 \mathrm{~cm}$ subtending 3.3degrees of visual angle. Lexical distractor strings were of four types: Words, orthographically legal nonword strings, orthographically illegal nonword strings, or uniform letter strings and in addition to the lexical distractors, a non-lexical distractor string was included. Fifty-two high frequency four-letter words were selected for the word strings (e.g. LOST). Fifty two orthographically legal nonword strings (e.g. LUPT), and fifty two orthographically illegal nonword strings (e.g. LGNT) were also constructed. Uniform letter strings were created (e.g. AAAA) from each of the letters of the alphabet other than I. Finally, a uniform shape string, comprised of a sequence of four boxes $(\square \square \square \square$ ) that were similar in size to the letter strings was constructed. The uniform shape string distractor was repeated 26 times at each eccentricity in the experiment.

\section{Design}

Two files of trials, each made up of 5 blocks of 104 randomly ordered trials were presented in a counterbalanced order across participants such that peripherally presented distractors appeared in the left hemifield as frequently as they appeared in the right hemifield. A within subjects design was employed with two independent variables, Eccentricity (central or peripheral distractor presentation), and Distractor Type (word, orthographically legal nonword, orthographically illegal nonword, uniform letter string or uniform shape string). There was also a single target control condition in which no distractor was present. In this condition the target also appeared 8 degrees to the left or the right of the midline. Eye movement latencies (SOL's) and error rates were recorded and analysed.

\section{Procedure}

The experiment was run on a Macintosh Quadra 700 computer with a Macintosh 21-inch screen operating at $76 \mathrm{~Hz}$. In house software displayed a central fixation target for a fixed period of 1 second ( 0.6 deg black square on a white background) designed to ensure participants fixated the centre of the screen prior to the start of each trial. This was followed by the stimulus display, which initiated eye-movement recording for 1 second, followed by a blank screen for 1 second. Each display contained a target cross, either on its own or with a distractor. Preceding each block of trials participants performed a calibration procedure consisting of nine points in a square grid, each of which had to be fixated sequentially. A practice block (20 trials) was completed prior to recording, followed by the 5 experimental blocks with breaks between each block. Participants were instructed to look at the cross as quickly as possible, and ignore any other stimuli appearing in the displays. A fixed level of screen brightness and contrast were used for all experiments.

\section{Results}

For these data and data for all subsequent experiments a semi-automated procedure was used to analyse the eye movement data. The first saccade was detected automatically using a velocity criterion of $30 \mathrm{degrees} / \mathrm{sec}$ and each record was inspected individually to check that the program had 'picked up' the appropriate saccade. The 
latency, amplitude and direction of each saccade were obtained.

\section{Excluded Data}

Any trials in which tracker loss occurred were excluded from analyses, as were trials where saccade onsets were outside the range of $100 \mathrm{~ms}-500 \mathrm{~ms}(6 \%$ of trials).

\section{Directional Errors}

Directional errors were also excluded from the analyses of eye movement onset latencies. In all cases where a directional error was made, a corrective second saccade to the actual target was invariably also made during the trial presentation. Errors were only made for the condition where a peripheral distractor was presented, and therefore, the calculation of error rates did not include trials where either central distractors were presented or those trials where no distractor was presented with the target. Two participants were excluded from the analyses completely on the basis of making greater than $25 \%$ errors.

The mean error rates for the different Distractor Types were: words - 22\%, orthographically legal letter strings - $26 \%$, orthographically illegal letter strings $26 \%$, uniform letter strings - $23 \%$ and uniform shape strings - $22 \%$. Aone way (Distractor Type) ANOVA for the eccentric distractors was carried out on the mean error rates treating participants as a random variable. This revealed that there were no significant differences between the different Distractor Types $(F<1)$.

\section{Remote Distractor Effects}

Figure 1 shows the mean eye movement latencies for each condition. There was a highly reliable RDE. All t's $>10$, all p's $<.0001$, with saccade latencies being longer for all conditions where a distractor was present (mean $227 \mathrm{~ms}$ ) compared with the condition where no distractor was present (mean $180 \mathrm{~ms}$ ). The results are consistent with the expectation that all distractors would produce longer eye movement latencies compared to the control condition where no distractor was presented with the target, and this is seen clearly in Figure 1 where the control data for the no distractor condition is plotted. The magnitude of the RDE for central distractors was $53 \mathrm{~ms}$ and the magnitude of the RDE for peripheral distractors was $40 \mathrm{~ms}$.

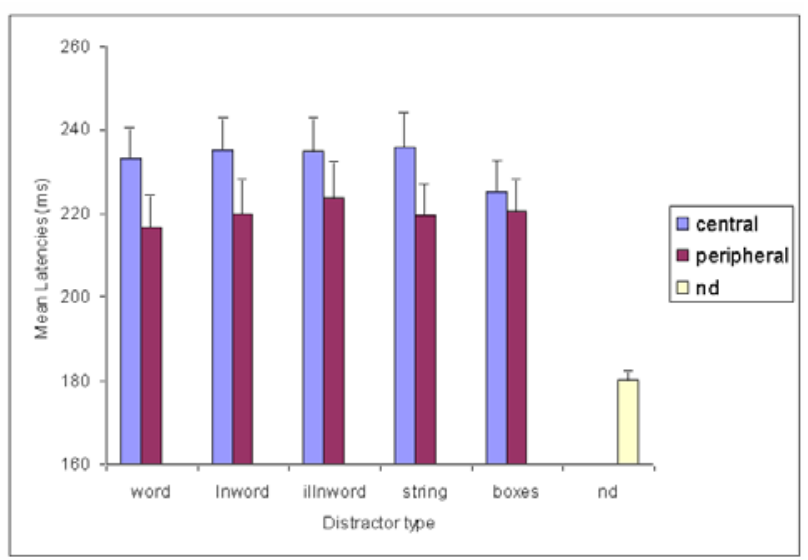

Figure 1: The mean saccade latencies for each of the five distractor types plotted for each of the two distractor eccentricities, with, additionally, the mean for the no distractor condition. Error bars denote 1 standard error from the mean.

\section{Saccade latencies}

A repeated measures ANOVA with Eccentricity (central and peripheral) and Distractor Type (word, legal nonword, illegal nonword, uniform letter string and uniform shape string) was conducted on the means for the correct eye movement latencies. There was a main effect of Eccentricity $(F(1,19)=17.07, p<.001)$ with centrally presented distractors (mean $=233 \mathrm{~ms}$ ), producing longer saccade latencies than distractors presented at the peripheral location (mean $=220 \mathrm{~ms}$ ).

A main effect was also found for Distractor Type ( $F$ $(4,76)=3.93, p<.01)$, which indicated that there were different effects upon saccade latencies between the different types of distractors. Paired $t$-tests showed that the non-lexical uniform shape string (boxes) distractor produced shorter saccade latencies $($ mean $=223 \mathrm{~ms}$ ) compared to the uniform letter string (mean $=228 \mathrm{~ms}), t$ $(1,39)=-2.2, \quad p<.05)$, the orthographically illegal nonword $($ mean $=229 \mathrm{~ms}), t(1,39)=-4.2, p<.001)$, and the orthographically legal nonword (mean $=228 \mathrm{~ms}$ ), $t$ $(1,39)=-2.7, p<.01)$. There was no difference between the non-lexical distractors and the word distractors (mean $=225 \mathrm{~ms}), t<1, p>$. 1 .

The main effects were qualified by an interaction between Distractor type and Eccentricity $(F(4,76)=$ $4.15, p<.005)$. Analyses revealed significant differences between central and peripheral presentation for all types of lexical string distractors (all t's $>3.7$; $p$ 's $\leq .001$ ) but not for the non-lexical uniform shape string (boxes) $(t$ $(1,19)=1.3, p>.1)$. This was an unexpected finding 
since the original RDE studies (Walker et al., 1997) showed that central distractors always produced a greater $\mathrm{RDE}$ compared to peripheral distractors.

No significant differences were found between the different lexical distractor types for central presentation of the distractors (all $t$ 's $<1.1 ; p$ 's $>1$ ). However at the peripheral presentation a difference between the orthographically illegal distractor string and all the other types of distractor strings was obtained (all $t$ 's $>2$; $p$ 's $\leq .05$ ). Because it was not predicted that an effect of orthographic status of the lexical strings would be observed at the peripheral presentation, a one-way ANOVA was computed on the peripheral data only. This analysis revealed that there were no significant differences between the different types of distractor at peripheral locations $(F<1)$.

\section{Discussion}

The data clearly demonstrate a RDE for all types of distractor string at both eccentricities. Additionally, Experiment 1 showed a number of other interesting effects including a clear eccentricity effect for lexical strings, but no such effect for the non-lexical shape string, and no systematic differences in saccade latencies between different types of centrally presented lexical distractor strings.

The absence of any effect of the lexical nature of the letter strings might be interpreted as indicating that lexical processing did not impact upon the system responsible for saccadic orienting, perhaps suggesting that the RDE is simply dependent on the visual stimulation caused by the distractor. However, if this was the case a similar RDE might be expected for the nonlexical as for the lexical distractor string. It could be that at central location a ceiling effect occurred for lexical stimuli. At central presentation automated lexical processing procedures were initiated and, either discontinued or completed extremely quickly for all types of lexical distractors. It has been shown that centrally presented lexical stimuli are identified as words or nonwords with relative ease (e.g., Meyer \& Schvaneveldt, 1971).

The lack of RDE differences between lexical distractor strings at eccentric locations most likely occurred because participants were unable to extract sufficiently detailed visual information to allow discrimination between them. This fits with the findings that, at least during the reading of text, only low-level visual information is available to the language processing system outside the parafovea (see: Rayner \& Pollatsek, 1989).

Saccade latencies for the non-lexical distractor strings at central and peripheral locations were not reliably different from each other. Close consideration of the design revealed an important difference between distractors in the lexical and non-lexical conditions, other than their differential lexical status; namely, the nonlexical distractor string comprised a set of four squares that was repeated across all trials. By contrast, the lexical distractors were made up of differing constituent letters and therefore their identity changed across trials. Consequently, the reduced RDE for non-lexical distractors at central locations might have occurred either as a consequence of their non-lexical status, or alternatively, as a consequence of their repetition. If the former conclusion is correct, then it suggests that lexical identification procedures were initiated automatically and did affect saccade onset decisions. Alternatively, if the latter explanation is correct, then the prediction is that the repeated presentation of a stimuli should lead to a diminished distractor effect, possibly via some form of visual learning mechanism. There is some evidence from visual search indicating that repetition of distractor presentation can decrease saccade latencies (Nakayama, Maljkovic \& Kristjansson, 2004). These possible explanations were tested in Experiment 2.

\section{Experiment 2}

Experiment 2 employed similar stimuli to those in Experiment 1 and manipulated three variables: the lexical status of the distractor; whether the distractor was repeated or changed across trials; and the eccentricity of the distractor. Both the uniform non-lexical string and the uniform letter strings from Experiment 1 were used in Experiment 2. However, rather than having a single uniform non-lexical string (as in Experiment 1), a new set of uniform non-lexical strings was created comprising 26 different strings each with 4 identical constituent shapes. These will be referred to as uniform shape strings.

The two types of distractor were therefore, lexical, made up of uniform letter strings (e.g. AAAA, BBBB 
etc.) or non-lexical, made up of uniform shape strings (e.g. $\checkmark \checkmark \checkmark, 2 \mathcal{D 2}$ ). Additionally, in each set of changing distractors there was one repeated distractor, which was from the alternative distractor set. The uniform shape string distractor set had 25 changing uniform shape strings plus one repeated (25 repetitions) uniform letter string, chosen randomly from the full set of 26 uniform letter strings. The uniform letter string distractor set had 25 changing uniform letter strings plus one repeated (25 repetitions) uniform shape string, chosen randomly from the full set of 26 uniform shape strings.

\section{Method}

\section{Participants}

16 members of the University of Durham community participated in the experiment.

\section{Materials}

As in Experiment 1 all characters for the lexical distractor strings were presented in upper case. They were created using Microsoft Sans Serif font size 24 point, overall length approximately $3.8 \mathrm{~cm}$, subtending 3.3 degrees of visual angle. All distractor strings were four uniform letters or four uniform symbols. The uniform shape strings were comprised of either four shapes or four symbols of a similar size to the letter strings and none of these distractors had lexical content. In Block A distractors comprised 25 different uniform letter strings and 1 constant (repeated) shape string (uniform shape string $\square \square \square \square$ from Experiment 1). In Block B distractors were 25 different uniform shape strings and 1 constant (repeated) letter string (HHHH), which was randomly selected from the set of uniform letter strings used in Experiment 1. Order of Block was counterbalanced across participants and the task and sequence of presentation was the same as in Experiment 1.

\section{Design}

The design was within participants with three independent variables; Constancy (changing distractor or repeated distractor), Eccentricity (central distractor or peripheral distractor) and Distractor Type ( letter string or shape string). Dependent variables were onset latencies and directional errors.

\section{Procedure}

Stimuli were displayed on a Philips 21B582BH 21 inch monitor at a viewing distance of $67 \mathrm{~cm}$. The monitor had a $\mathrm{P} 22$ phosphor with a decay rate to zero of less than 2 milliseconds. The monitor and the eyetracker were both interfaced with a Philips Pentium III PC that controlled the experiment. Successful calibration was followed by two experimental blocks of trials (400 trials in each).

\section{Results}

\section{Excluded Data}

Data excluded from analyses included trials in which tracker loss occurred and trials where saccades were outside the range of $100 \mathrm{~ms}-500 \mathrm{~ms}$. ( $8 \%$ in total).

\section{Directional Errors}

Directional errors were also excluded from the main analyses of eye movement onset latencies. For changing stimuli the mean error rate for letter strings was $19 \%$, and for shape strings $15 \%$. For the repeated distractors the mean error rate for letter strings was $19 \%$ and for the shape strings $14 \%$. A 2(Constancy) X 2(Distractor Type) repeated measures ANOVA was performed on the mean error rates for peripheral distractor trials treating participants as a random variable. There was a significant main effect of Distractor Type $(F(1,15)=$ $8.25, \mathrm{p}<.05)$, no significant effect of Constancy, and no interaction between the two, (F's < 1). A higher proportion of errors were made when the distractors were made up of letters, compared to those made from shapes.

\section{Remote Distractor Effects}

A reliable $\mathrm{RDE}$ (all $t$ 's $>9$, all $p$ 's $<.0001$ ) was observed for all conditions, with increased saccade latencies for distractor present trials (single target mean saccade onset latency $=171 \mathrm{~ms}$ ). The mean saccade latencies for the distractor present trials are presented in Figure 2. 
Panel (a)

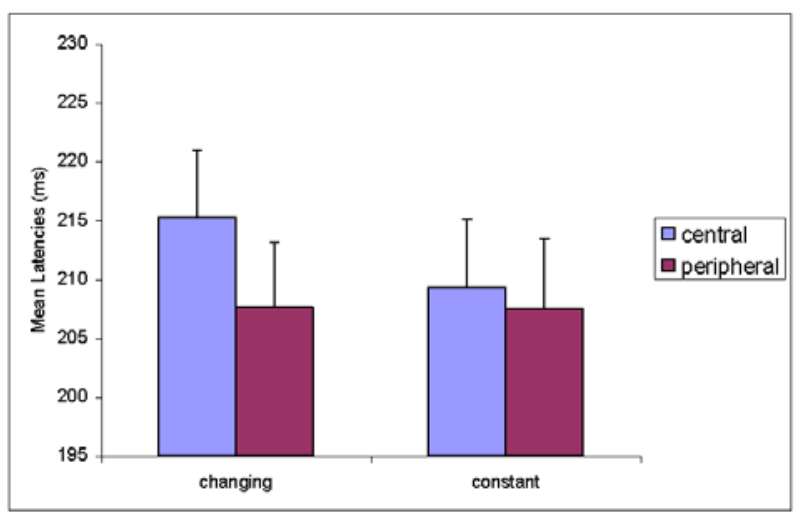

Panel (b)

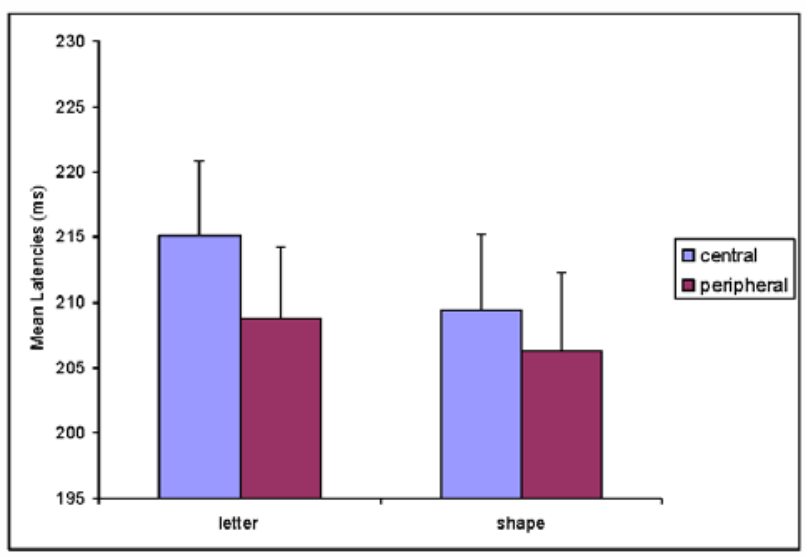

Panel (c)

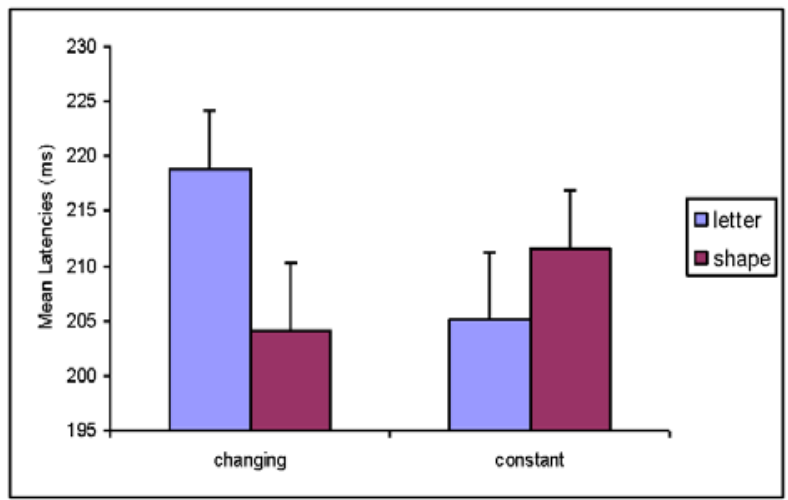

Figure 2:Panel (a). The mean saccade latencies for changing and repeated distractors for each of the distractor eccentricities. Panel (b). The mean saccade latencies for letter string and shape string distractors for each of the distractor eccentricities. Panel (c). The mean saccade latencies for changing and repeated distractors for uniform letter strings, and uniform shape strings. Error bars denote 1 standard error from the mean.

\section{Saccade latencies}

A 2(Eccentricity) X 2(Constancy) X 2(Distractor Type) ANOVA was conducted on the mean correct saccade latencies treating participants as a random variable. A main effect of Constancy $(\mathrm{F}(1,15)=8.05$, $\mathrm{p}$ $<.05)$ showed that saccade onsets were longest when distractors were changing (mean $=211 \mathrm{~ms}$ ) across trials, compared to when they were repeated $($ mean $=208 \mathrm{~ms}$ ) across trials. There was also a main effect of Eccentricity $(\mathrm{F}(1,15)=4.59, \mathrm{p}<.05)$, with saccade latencies being significantly longer when distractors were presented centrally (mean $=212 \mathrm{~ms}$ ) compared to when they were presented peripherally (mean $=208 \mathrm{~ms}$ ). This result is in line with the typical finding from Walker et al.'s (1997) RDE investigation. There was also a reliable effect of Distractor Type $(\mathrm{F}(1,15)=10.17, \mathrm{p}<.01)$. Saccade latencies were longer for the uniform letter string distractors (mean $=212 \mathrm{~ms}$ ) than for the uniform shape string distractors (mean $=208 \mathrm{~ms}$ ), regardless of whether they were repeated or changing. This suggests that increased saccade latencies were associated with the lexical status of the distractors.

The main effects of Constancy, Eccentricity and Distractor Type obtained in Experiment 2 were, however, qualified by interactive effects. There was a reliable interaction between Constancy and Eccentricity $(F(1,15)$ $=5.4, p<.05$ ). Panel (a) of Figure 2 shows the mean saccade latencies for changing and repeated distractors at central and peripheral locations. Central presentation produced longer saccade latencies compared to peripheral presentation $(\mathrm{t}(1,31)=3.71, p<.001)$ for changing, but not for repeated $(t<1)$ distractors. This result is consistent with the finding for the repeated distractor in Experiment 1.

The interaction between Eccentricity and Distractor Type was marginal $(F(1,15)=4.0, p=.06)$. Panel $(b)$ of Figure 2 shows that the eccentricity effect for letter strings was numerically greater than that for shape strings. If the eccentricity effects observed for lexical distractors but not the uniform shape distractor in Experiment 1 arose as a consequence of their lexical status, the interaction between Distractor Type and Eccentricity should arise due to a difference in saccade latencies between central and peripheral uniform letter strings, but not for uniform shape strings. Longer saccade latencies occurred for the uniform letter strings at central presentation compared to peripheral presentation 
$(\mathrm{t}(1.31)=3.26, \mathrm{p}<.01)$. However for the uniform shape strings there was no reliable difference between central and peripheral distractor locations $(\mathrm{t}(1.32)=1.26, \mathrm{p}>$ .1). The nature of this interaction is also consistent with the effects observed in Experiment 1 and the finding indicates that the lexical status of a distractor string has an increased effect when presented at central compared to peripheral retinal locations. No similar effect was observed for uniform shape strings. Arguably, the increased saccade latencies associated with letter string distractors at central locations reflect lexical processing times that do not occur either for shape strings, or for letter strings that are visually degraded due to acuity limitations associated with peripheral processing.

There was a reliable interaction between Constancy and Distractor Type $(F(1,15)=5.3, p<.05)$. Panel (c) of Figure 2 shows the mean saccade latencies for uniform letter string distractors and uniform shape string distractors under changing and repeated conditions. Longer saccade latencies were produced for the letter strings when these were changing compared to when they were repeated $(t(1,31)=3.47, p<.001)$. This result is consistent with the suggestion of greater saliency for changing than for repeated distractors. However, the comparison for the uniform shape strings revealed that longer saccade latencies occurred when they were repeated compared to when they were changing, $(t(1,32)$ $=-2.31, p<.05)$. Note that although the effect for the shape strings was weaker than for the letter string distractors, the direction of the effect was still unexpected. Finally, there was no reliable three-way interaction between Constancy, Eccentricity and Distractor Type $(\mathrm{F}<1)$.

\section{Discussion}

Experiment 2 investigated whether the eccentricity effects observed in Experiment 1 arose as a consequence of the lexical status, or the repeated nature of the distractor strings. The main effects clearly demonstrate that saccade latencies were affected by retinal eccentricity of the distractor, lexical status of the distractor, and whether distractors were repeated or changing across blocks of trials. To this extent these findings highlight the importance of each of these variables in relation to the computation of saccadic onsets in the RDE paradigm. The finding that distractor repetition has an effect on saccade latencies reproduces the effect found in Experiment 1. This is thought to be a novel result, although similar to findings in visual search (Nakayama, Maljkovic \& Kristjansson, 2004) showning that items that are repeated during the course of the experiment do not attract attention/saccades to the same degree as novel items (Johnston, Hawley, \& Farnham, 1993; Johnston, Hawley, Plewe, Elliot \& De Witt, 1990).

The interactive effects indicate that these variables have a combined impact on the saccade generation system in the RDE paradigm. Changing remote distractors produced the predicted increase in saccade latencies for central compared to peripheral presentation. This effect was absent for repeated distractors and occurred regardless of whether the distractor string was a uniform shape or a uniform letter string. It appears that there is sensitivity on the part of the visual processing system to the frequency with which a visual distractor is encountered, and that increased exposure to the same stimulus results in a reduction of its visual saliency as a distractor. These results imply that any remote distractor that is repeated across experimental trials, at least at central presentation, attains sufficient familiarity during the extended course of an experiment as to render it less visually salient than counterpart unrepeated (relatively novel) stimuli. The finding suggests that some form of learning mechanism exists which impacts upon the oculomotor control system.

The marginal interaction between Eccentricity and Distractor Type was also in line with the findings in Experiment 1 and supports the suggestion that the eccentricity effects observed in Experiment 1 resulted directly from the lexical distractors producing a processing cost at central presentation that was absent for the uniform shape strings. Together, these two interactions indicate that the lexical status of a distractor string, as well as whether it is repeated or changing across trials, both impact upon the effectiveness of the distracting influence (at least at foveal locations).

A further interesting finding was that the difference between changing and repeated distractors was smaller for uniform shape strings than for uniform letter strings and that the effect was in the opposite direction for uniform shape strings than for uniform letter strings. Why should repeated uniform shape strings produce increased onset latencies compared to changing shape strings in this experiment? One explanation is that the 
latencies for the repeated shape strings could have been affected by this distractor being presented amidst a set of changing letter strings. It is possible that there was some carryover effect from the cost in terms of saccade latencies for processing the changing uniform letter strings, that manifested in an increase in saccade latencies for the repeated distractor that was embedded in that set of distractors. In the same way, it is also feasible that there was some carryover effect from processing the changing uniform shape strings that produced a decrease in saccade latencies for the repeated letter distractor in that block. Such carryover effects would indicate that differences in processing times for a particular type of changing distractor (uniform letter strings versus uniform shape strings) impact in a systematic way upon the saccadic reaction time for a repeated distractor that is presented during the same block of trials. This finding in itself provides some evidence for complex distractor effects in the RDE paradigm.

\section{Experiment 3}

Experiment 3 investigated whether repeated distractors embedded within changing distractors of the same category would still produce a reduced effect, and whether a parafoveal distractor presentation location would produce systematic effects for the type of distractor presented (lexical and non-lexical).

In Experiment 3, therefore, for each block of trials, participants were presented with distractor strings (similar to those used in Experiment 1), that contained changing items and one repeated item from the same category. In order to ensure that the number of conditions in Experiment 3 remained manageable, three categories of distractor were employed; words, orthographically illegal strings and shape strings (e.g. 'TALK', 'PVTK', and ' $\square \triangleright \bigcirc \Delta$ ' respectively). Words and orthographically illegal nonwords were selected for this experiment as these two types of lexical string differ most from each other in terms of their lexical status thereby optimising the possibility of obtaining differential modulation of the RDE. The shape string distractors were made up of four different shapes (in the same way that each lexical string was made up of four different letters). This was to ensure that all the (lexical and shape) distractors were comparable, at least in terms of comprising four unique sub-units.

The inclusion of an intermediate distractor eccentricity (4 degrees eccentric from the display midline) was to test the possible hypothesis that ceiling effects were observed in Experiment 1 for the lexical string distractors. Since visual acuity drops off from central to peripheral eccentricities, extraction of visual information at intermediate points between such locations should be slowed, but not so reduced that visual discrimination between qualitatively different stimuli would be impossible. Hence, it was anticipated that any subtle processing differences may be more likely to manifest at this presentation location.

It was predicted that repeated distractors would produce shorter saccade latencies compared to changing distractors, lexical distractors should result in longer saccade latencies compared to the shape string distractors, and a difference would be obtained between the two types of lexical distractor at the intermediate distractor presentation location (but not at central or peripheral locations).

\section{Method}

\section{Participants}

15 members of the University of Durham community participated in Experiment 3.

\section{Materials}

As in Experiment 2 all characters for the lexical distractor strings were presented in upper case using Microsoft Sans Serif font size 24 point, overall length approximately $3.8 \mathrm{~cm}$, subtending 3.3 degrees of visual angle. The symbol strings were comprised of either four shapes or four symbols of a similar size to the letter strings with no lexical content. Letter string distractors were either four letter high frequency words or four letter orthographically illegal letter strings and were taken from the same set of letter string distractors used in Experiment 1. A total of 26 different distractor strings were created for each of the three different types of distractor string (word, illegal nonword and shape). One string from each type was selected randomly to appear as the repeated distractor, leaving 25 distractor strings from each type to appear as the changing distractors. The 
repeated distractor for the word distractor condition was (JUMP), the repeated distractor for the orthographically illegal nonword condition was (RFMP), and the repeated distractor for the shape condition was the following sequence of symbols ( $\triangle \cup \square \&$ ). In total there were 25 changing and 25 repeated distractors at each of the three eccentricities with the target positioned at left or right of the display at either 4 degrees eccentricity or 8 degrees eccentricity. In each block of trials there were therefore 400 trials for each condition where a distractor was presented simultaneously with a target and there were 100 trials where a target was presented on its own (25 times at Left and Right for the two target eccentricities of 4 and 8 degrees).

\section{Design}

The design of the Experiment was within participants with three independent variables; Constancy (2 levels; changing distractor or repeated distractor), Eccentricity (3 levels; central distractor or peripheral distractor at 4 degrees or peripheral distractor at 8 degrees) and Distractor Type (3 levels; four letter word, four letter orthographically illegal nonword and four symbol shape string). In Block A distractors were words. In Block B distractors were orthographically illegal nonwords. In Block C distractors were shape strings. Order of block was counterbalanced across participants.

\section{Procedure}

The calibration procedure, sequence of trial presentation and participant instructions were the same as in the previous experiments.

\section{Results}

\section{Excluded Data}

Data excluded from analyses included Tracker Loss trials $(5.3 \%)$ and trials where correct saccades to target were initiated either before $100 \mathrm{~ms}$ or after $500 \mathrm{~ms}(0.4 \%)$.

\section{Directional Errors}

Directional errors were also excluded from the final analysis of eye movement onset latency. As in Experiments 1 and 2 no directional errors were made in the central distractor presentation condition. A 2(Constancy) X 2(Eccentricity) X 3(Distractor Type) ANOVA was carried out on the mean error rates treating participants as random variables. There was a significant main effect of Eccentricity $(F(1,14)=19.74, p=.001)$, with participants making more errors when the distractor was at the 8 degree location (mean $=28 \%$ ) than when it was at the 4 degree location (mean $=20 \%$ ) and no other significant effects. More errors were made when the distractor was positioned at the far peripheral location of 8 degrees eccentricity. It is suggested that in this case the peripheral distractors have an effect that is similar to that observed in the oculomotor capture paradigm (e.g. Theeuwes, Kramer, Hahn, \& Irwin, 1998; Theeuwes, Kramer, Hahn, Irwin, \& Zelinsky, 1999), but there is no evidence for any particular type of distractor producing more errors than another.

\section{Remote Distractor Effects}

There was a highly reliable Remote Distractor Effect (all $t$ 's $>3.5$, all $p$ 's $<.005$ ), with saccade latencies being longer for all conditions when a distractor was present compared with the single target condition (No distractor mean saccade onset latency $=182.0 \mathrm{~ms}$ ).

\section{Saccade latencies}

A 2(Constancy) X 3(Eccentricity) X 3(Distractor Type) ANOVA was performed on the mean saccade latencies for correct responses treating participants as random variables. There was a small but reliable main effect of Constancy $(F(1,14)=9.10, p<.01)$ with latencies being longest when distractors were changing (mean $=206 \mathrm{~ms})$ than when they were repeated (mean $=$ $205 \mathrm{~ms}$ ). This effect was very small and weaker compared to that shown in the previous two experiments (mean difference between changing and constant distractors Experiment $1=4.4 \mathrm{~ms}$, Experiment $2=3.1 \mathrm{~ms}$, Experiment $3=1.4 \mathrm{~ms}$ ) but it does seem to occur reliably in all experiments reported in this paper, and the reduced effect is possibly a result of the same category repeated and changing distractors being presented in the same block in this experiment, as opposed to cross category changing and repeated distractors being presented in the same block of trials in the previous two experiments. No other main effects were obtained ( $F$ 's $<2.4)$.

There was a reliable interaction between Eccentricity and Distractor Type $(F(4,56)=3.1, p<.05)$. The mean saccade latencies for each of the three distractor types at each of the three eccentricities are given in Figure 3 panel (a). At central presentation shape strings $($ mean $=201 \mathrm{~ms})$ 
produced significantly shorter saccade latencies than word $($ mean $=207 \mathrm{~ms})$ distractors $(t(1,14)=27.4, p<$ $.001)$ and orthographically illegal (mean $=206 \mathrm{~ms}$ ) distractors $(t(1,14)=19.8, p<.001)$, but there was no difference between the two types of lexical distractor, $F$ $<1$.

At peripheral presentation of 4 degrees shape strings (mean $=202 \mathrm{~ms})$ produced significantly shorter saccade latencies $(t(1,14)=91.5, p<.001)$ than words (mean $=$ $213 \mathrm{~ms})$ distractors $(t(1,14)=27.4, p<.001)$ and orthographically illegal (mean $=208 \mathrm{~ms})$ distractors $(t(1$, $14)=25, p<.001)$, and there was a difference between the two types of lexical distractor, $(t(1,14)=20.8, p<$ $.001)$ with words producing significantly longer saccade latencies at this presentation location than orthographically illegal strings.

At the far peripheral distractor presentation of 8 degrees the shape strings $($ mean $=198 \mathrm{~ms}$ ) produced significantly shorter saccade latencies than both the word distractors $(t(1,14)=58.2, p<.001)$, and the orthographically illegal distractors $(t(1,14)=13.4, p<$ $.001)$. Thus at all eccentricities the lexical distractors have greater disruptive effects upon saccade latencies, compared to the non-lexical shape string distractors. Additionally, words (mean $=207 \mathrm{~ms}$ ) resulted in greater saccade latencies than the orthographically illegal strings $($ mean $=203 \mathrm{~ms}),(t(1,14)=15.8, p<.001)$. This was unanticipated. However, although the two-way interaction between Eccentricity and Distractor Type has shown that word distractors appear to have a more disruptive effect upon saccade latency production, compared to nonword distractors at eccentricities well into the periphery, this finding is qualified by a three-way interaction that shows that this applies exclusively for the repeated nonword distractors and is discussed below.

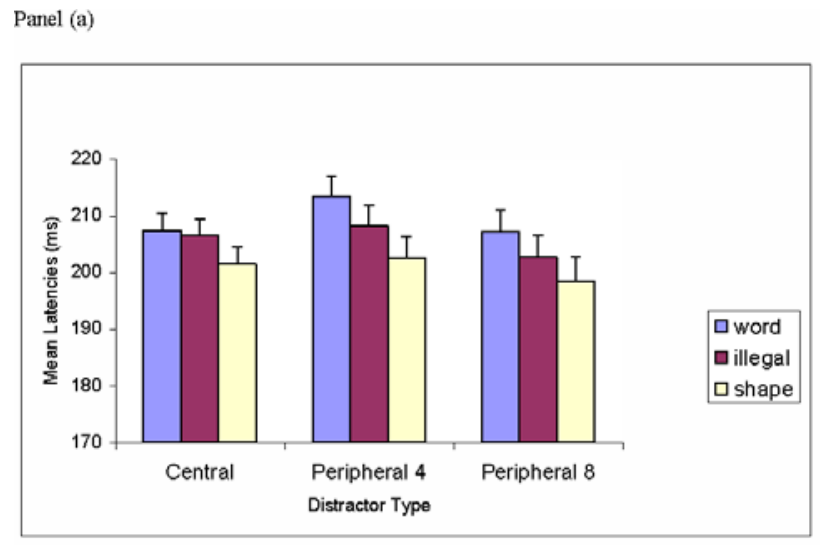

Panel (b)

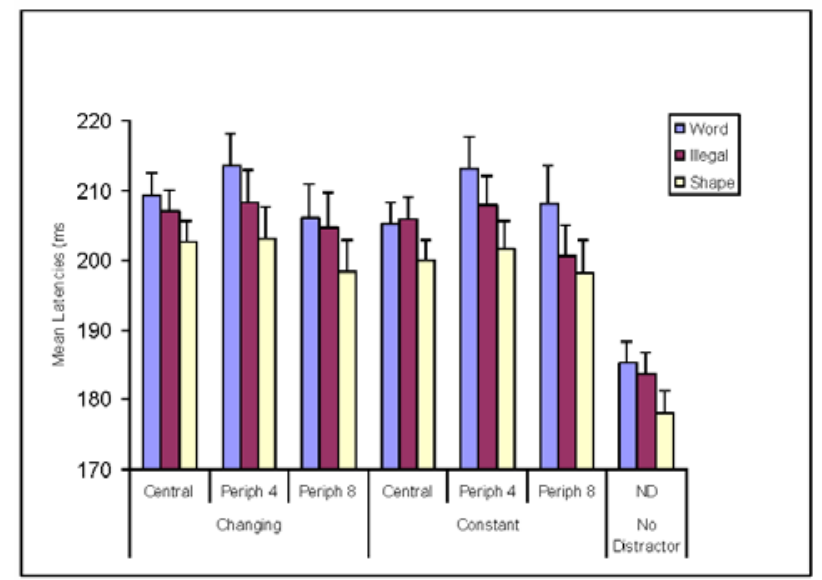

Figure 3: Panel (a). The mean saccade latencies for each of the three Distractor Types at each of the three Eccentricities in Experiment 3. Panel (b). The mean saccade latencies for each of the three Distractor Types, at each of the three Eccentricities, for both Changing and Repeated distractors. Error bars denote one standard error from the mean.

A three-way interaction between Constancy, Eccentricity and Distractor Type $(F(4,56)=3.7, p<.05)$ was obtained. The data corresponding to the three-way interaction are shown in Figure 3 panel (b). For all the comparisons except one, the expected pattern of saccade latencies was found with both the changing and repeated distractors. Namely, shape string distractors produced shorter latencies compared to the two types of lexical distractors, there were no differences between the two types of lexical distractor at central presentation, but words resulted in longer saccade latencies compared to the nonwords at an intermediate peripheral presentation. However for the 8 degree peripheral presentations the word vs. the orthographically illegal letter string difference was large and significant for the repeated 
presentations but small and non significant for the changing ones. This difference appears to have been consistent enough to generate the significant interaction. It is unlikely that this finding reflects a real difference between the two distractor types at this eccentricity, since this would not be supported, either by the findings for the 8 degree peripheral distractors in the previous experiments, nor would it be predicted by any theoretical assumption for this eccentricity. There is the possibility that chance design factors could have produced this result as the repeated word (JUMP) may have had attributes that made it more salient at the far peripheral distractor location, than would have been predicted. No items analyses were possible since each block contained both repeated and changing items, therefore this suggestion cannot categorically be ruled out.

\section{Single target trial data}

Figure 3 panel (b) also includes the data from the single target trials, separated by the type of distractor block, although these data were not included in the ANOVA reported above. The pattern observed for the trials where no distractor is presented with the target shows striking similarity to the overall pattern shown for each of the different distractor types. Namely, single target trials in the word distractor block $($ mean $=185 \mathrm{~ms})$ resulted in longer saccade latencies compared to the single target trials presented in the orthographically illegal distractor block (mean $=183 \mathrm{~ms}$ ), and the single target trials in the orthographically illegal distractor block produced longer saccade latencies than those produced for the single target trials in the shape string block (mean $=178 \mathrm{~ms}$ ). Although paired samples t-tests revealed that these numerical differences were not statistically reliable ( $t$ 's $<1.5$ ), the overall pattern is still interesting and suggests that differences caused by distractor stimuli can occur even when the distractors themselves are not actually present. This is also consistent with the explanation for the finding from Experiment 2 whereby an increase in saccade latencies was observed for the repeated shape distractor when it was presented amidst a set of changing letter string distractors, and supports the hypothesis that a carryover effect for the processing of the different distractor types results in increased latencies for the repeated shape strings and decreased latencies for the repeated letter string distractors in that experiment.

\section{RDE Magnitudes}

The design of Experiment 3 allowed an analysis that took possible carryover effects into account. Given that each of the three different types of distractor were presented individually in blocks of trials, and each block included a set of single target trials, it was possible to calculate corresponding single target trial latencies as a separate baseline for each distractor type. The RDE magnitude was calculated, on an individual participant basis, as the difference between the distractor present trials and the corresponding baseline single target trials. Table 1 shows the mean RDE magnitudes (Latency increase compared to the single target trials) for each distractor type separately.

Table 1: Mean RDE magnitudes in ms for Experiment 3 (with standard deviations in parentheses) for each Distractor Type.

Type of Distractor

\begin{tabular}{ccc} 
Word & Illegal & Shape \\
\hline 24.1 & 22.1 & 22.6 \\
$(13.7)$ & $(15.0)$ & $(5.7)$
\end{tabular}

A one way ANOVA for the variable Distractor Type, with three levels corresponding to each type of distractor was computed. This revealed that there were no significant differences between any of the three types of distractor on RDE magnitudes $(F<1)$. This important result modifies the conclusions that can be drawn from the present experiments and demonstrates that the difference between the three types of distractor (in terms of effects upon saccadic latencies) does not reflect a difference in RDE magnitudes for each distractor type.

\section{Discussion}

Experiment 3 had two aims. The first was to determine whether the repeated distractor effect found for Experiments 1 and 2 would hold if the changing and repeated distractors belonged to the same category of distractor. This was supported and a very small but highly reliable effect of constancy indicated that repeated 
distractors produced shorter saccade latencies compared to changing distractors. The repeated distractor effect has been found in all three experiments and is particularly important in Experiment 3 since it confirms that the effect is not specific to cross category distractor presentation. It can now be concluded that the repeated distractor effect is a robust emergent property that results from one stimulus type achieving the status of familiarity during the course of its presentation within a brief experimental session.

The second aim of the experiment was to see if the introduction of an intermediately located distractor between central fixation and the periphery would produce differential results for the different types of lexical distractors. There were plausible theoretical grounds for expecting that there might be differences between words and the orthographically illegal strings at the 4 degree distractor location, but based on the results of Experiment 1 , no differences were expected at central presentation or at the far peripheral presentation location of 8 degrees. The finding that words produced longer saccade latencies compared to illegal nonwords when presented parafoveally for both changing and repeated distractors suggests that words are processed in a qualitatively different way to illegal strings, at this eccentricity.

It was also predicted that the lexical distractors would produce a greater RDE compared to the shape string distractors. There was however no main effect of Distractor Type in this experiment. This is somewhat surprising given the reliable differences between the shape string distractors and the lexical distractors at all eccentricities for both changing and repeated distractors. Clearly, the lexical distractors produce longer saccade latencies. However a separate analysis of the RDE magnitudes for each distractor type revealed that all types of distractor produced equivalent RDE magnitudes. This suggests that any RDE modulating influences may be independent of saccade latency influences when examining the influence of complex distractors. Finally, it appears that systematic influences upon saccade latencies for single target control trials result from the type of distractor that they are presented with.

\section{General Discussion}

The aim of these experiments was to examine possible influences of complex distractors upon the RDE.
Distractors were letter strings or strings of abstract symbols and were employed on the basis that letter strings, particularly familiar words, would be automatically processed (Stroop, 1935) and as such it was hypothesized that this processing would influence the saccade latencies in the RDE paradigm.

In all the experiments and for every observer tested, the latency of a saccade to a well defined target was increased when a distractor was simultaneously presented. Distractors presented centrally resulted, almost always, in a greater RDE than when the distractor was presented in the contralateral hemifield at a mirror symmetrical position.

A number of other effects were observed. In Experiment 1 abstract shape strings generated a smaller RDE than the letter strings at central presentation. Experiment 2 tested whether this difference resulted from the stimulus property differences between the types of distractors, or from the fact that one type of distractor was presented repeatedly. Lexical distractors generated greater interference than shape string distractors, but there was also an effect of distractor repetition. Distractors repeated regularly during the course of a block resulted in a reduced RDE at central presentation. This finding was followed up in Experiment 3, where repeated distractors were presented amidst changing distractors of the same class (word, letter string, or shape). Repeated distractors were again found to result in a reduced RDE compared to changing distractors, and although the difference observed in Experiment 3 was small, it was still highly significant. The repeated distractor effect is an important finding, since it suggests that there is some implicit learning of the repeated item during testing that makes it more discriminable from the changing items. Whether this occurs in a completely automatic fashion, or is a result of some sort of strategic learning remains to be addressed. Implicit learning has been shown to influence eye movements in several ways in the visual search and attention literature (McPeek, Maljkovic and Nakayama, 1999, Peterson \& Kramer, 2001; Lambert, Norris, Naikar \& Aitken 2000). The effect for repeated distractors is similar to that obtained in the 'priming of pop-out' studies, in that, saccade latencies decreased for repeated distractors. This suggests that the effect may be a consequence of an automatic learning mechanism similar to that responsible for 'priming of pop-out' and may serve to free up attentional processing 
resources during visual tasks. Current models of oculomotor control do not, at present, offer any explanation for the repeated distractor effect although Findlay and Walker's (1999) model does allow for quick and direct cognitive influences on decisions concerning when to move the eyes. The model specifies that the processes operating at this level are automated and the role of implicit learning and memory may be significant at this level, though no mechanistic account for how this actually occurs is given.

In Experiment 3, at all distractor locations, consistent differences in latencies were found with different distractor types. Latencies were longest with word distractors and illegal letter strings, and shortest with shapes. Additionally, at parafoveal presentation, a latency difference was found for the first time between words and orthographically illegal letter strings. This finding fits in some ways with the findings from the reading literature that show that parafoveal orthographic information is extracted and used in the guidance of saccades (e.g. White \& Liversedge, 2005). It also shows that complex distractors can have systematic influences upon saccade latencies in the RDE paradigm that may be directly related to the specific nature of the distractor, and how that type of distractor is processed in other tasks.

An unexpected finding was that the latencies on single target trials were affected by the material presented within the block. This new finding of block-wise changes in latency has both theoretical and methodological implications. The blocked design allowed a separation of saccade latency from RDE magnitude, for each type of distractor. When both measures were analysed, it emerged that RDE magnitudes were unaffected by type of distractor. It is important to note that this does not mean that the latency differences between the different types of distractors are unimportant in these experiments; they clearly demonstrate a highly reliable influence of complex distractors affecting the saccade generating system. The differential pattern of effects for saccade latencies and RDE magnitudes could reflect two distinct phenomena. The RDE of constant magnitude observed for all types of distractor reflects a fixed, reflexive aspect of oculomotor behaviour that occurs whenever a distractor appears synchronous with a target. By contrast, the variable effect observed for saccade latencies reflects a more long-lasting, pervasive influence directly related to the type of stimulus (word, nonword, shape string) that is presented as a distractor. Furthermore, this influence not only affects latencies when a distractor is present, but also exerts an influence for single target control trials in that block. The differential distractor type effects for saccade latencies and RDE magnitudes is interesting as it suggests that although there may be complex distractor influences on saccade latencies in this paradigm, these saccade latency influences do not impact upon RDE magnitudes. As such, and in conclusion, these finding support the claim that the RDE reflects automated, reflexive oculomotor processing (Walker et al., 1997).

\section{References}

Coltheart, M., Davelaar, E., Jonasson, J.T., \& Besner, D. (1977). Access to the internal lexicon. In S. Dornic (Ed.), Attention and performance, VI (pp. 535-555). New York: Academic Press.

Findlay, J. M., \& Walker, R. (1999). A model of saccade generation based on parallel processing and competitive inhibition. Behavioral and Brain Sciences, 22, 661-674

Godijn, R. \& Theeuwes, J. (2002). Programming of exogenous and endogenous saccades: Evidence for a competitive integration model. Journal of Experimental Psychology: Human Perception and Performance 28 (5): 1039-1054.

Johnston, W.A., Hawley, K.J., \& Farnham, J. (1993). Novel popout: Empirical boundaries and tentative theory. Journal of Experimental Psychology: Human Perception and Performance, 19, 140-153.

Johnston, W.A., Hawley. K.J., Plewe, S.H., Elliot, J.M.G. \& De Witt, M.J. (1990). Attention capture by novel stimuli. Journal of Experimental Psychology: General, 119, 397-411.

Lévy-Schoen, A., (1969). Determination et latence de la response oculomotrice a deux stimulus simultanes ou successifs selon leur excentricite relative. A Psychol., 69, 373-392.

Lambert, A., Norris, A., Naikar, N.\& Aitken, V, (2000). Effects of informative peripheral cues on eye movements: Revisiting William James' “derived attention”. Visual Cognition., 7, (5), 1 545-569(25)

Liversedge, S.P. \& Findlay, J.M., (2000). Eye movements reflect cognitive processes. Trends in Cognitive Sciences, 4, 6-14. 
MacLeod, C. M. (1991). Half a century of research on the Stroop effect: An integrative review. Psychological Bulletin, 109, 163-203.

McPeek, R. M., Maljkovic, V., and Nakayama, K. (1999). Saccades require focal attention and are facilitated by a short-term memory system. Vision Research, 39(8), 1555-1566.

Meyer, D.E. \& Schvaneveldt, R.W., (1971). Facilitation in recognizing pairs of words: Evidence of a dependence between retrieval operations. Journal of Experimental Psychology, 90, 227-235.

Nakayama K, Maljkovic V, Kristjansson A., (2004). Short-term memory for the rapid deployment of visual attention. In Gazzaniga, M.S., (Ed.) The Cognitive Neurosciences III, 397-408.

Oliver, E., Dorris, M. C. and Munoz, D. P. (1999) Lateral interactions in the superior colliculus, not an extended fixation zone, can account for the remote distractor effect. Behavioral and Brain Sciences, 22, 694-695.

Peterson, M. S., Kramer, A. F. (2001). Contextual cueing reduces interference from task-irrelevant onset distractors. Visual Cognition, 8, 843-859.

Rayner, K. (1998). Eye movements in reading and information processing: 20 years of research. Psychological Bulletin, 124, 372-422.

Rayner, K. and Pollatsek, A. The psychology of reading. Prentice-Hall, 1989.

Rayner, K., Well, A.D., \& Pollatsek, A., (1980). Asymmetry of the effective visual field in reading. Perception \& Psychophysics, 27, 537-544.

Stroop, J.R., (1935). Studies of interference in serial verbal reactions. Journal of Experimental Psychology, 28, 643-662.

Sumner, P, Adamjee, T. and Mollon, J.D. (2002) Signals invisible to the collicular and magnocellular pathways can capture visual attention. Current Biology, 12, 1312-1316.

Theeuwes, J, Kramer, A.F, Hahn, S. \& Irwin, D. E. (1998). Our eyes do not always go where we want them to go: capture of the eyes by new objects. Psychological Science, 9, 379-385.
Theeuwes, J. Kramer, A.F., Hahn, S., Irwin, D.E. \& Zelinsky, G.J. (1999). Influence of attentional capture on oculomotor control. Journal of Experimental Psychology: Human Perception \& Performance, 25, 1595-1608.

Walker, R. Deubel, H. Schneider, W.X. \& Findlay, J.M. (1997) Effect of remote distractors on saccade programming: evidence for an extended fixation zone. Journal of Neurophysiogy, 78, 2, 1108-1119.

Walker, R. Kentridge, R.W. \& Findlay, J.M. (1995). Independent contributions of the orienting of attention, fixation offset and bilateral stimulation on human saccadic latencies. Experimental Brain Research, 103, 294-310.

White, S.J., \& Liversedge, S.P. (2004). Orthographic familiarity influences initial eye fixation positions in reading. European Journal of Cognitive Psychology, $16,52-78$. 